\title{
Common envelope evolution
}

\author{
Robert G. Izzard ${ }^{1}$, Philip D. Hall ${ }^{2}$, Thomas M. Tauris ${ }^{1,3}$ \\ and Christopher A. Tout ${ }^{2}$ \\ ${ }^{1}$ Argelander-Insitut für Astronomie, Universität Bonn, Auf dem Hügel 71, 53121 Bonn, \\ Germany \\ ${ }^{2}$ Institute of Astronomy, The Observatories, Madingley Road, Cambridge CB3 0HA, United \\ Kingdom \\ ${ }^{3}$ Max-Plack-Insitut für Radioastronomie, Auf dem Hügel 69, 53121 Bonn, Germany
}

\begin{abstract}
Many binary star systems are not wide enough to contain the progenitor stars from which they were made. One explanation for this is that when one star becomes a red giant a common envelope forms around both stars in the binary system. The core of the giant and its companion star continue to orbit one another inside the envelope. Frictional energy deposited into the common envelope may lead to its ejection and, if so, a close binary system is formed from the core of the former giant star and its relatively untouched companion. When the primary is an asymptotic giant branch star the core becomes a hot carbon-oxygen white dwarf which may ionise the ejected envelope and illuminate a planetary nebula. Many other types of binary systems form through common envelope evolution such as low-mass X-ray binaries and cataclysmic variables. In the case of a failed envelope ejection when the cores merge, rapidly-rotating solitary giants similar to FK Comae stars form. In this short review we focus on attempts to constrain parameters of common envelope evolution models and also describe the latest efforts to model this elusive phase of binary stellar evolution.
\end{abstract}

Keywords. binaries: general, stars: AGB and post-AGB, planetary nebulae: general, stars: winds, outflows

\section{The common envelope phase}

When stars grow old, they expand: this is a general result of stellar evolution (Iben 1993; Stancliffe et al. 2009). During shell-burning phases, such as the asymptotic giant branch (AGB), stellar radii reach sizes of several astronomical units. Many of these stars are in binary systems in which they orbit a dwarf companion and, if the binary separation is large enough, either star can pass through a red giant phase without significant interaction with its partner. If the stars are closer, however, the giant attempts to transfer material to its companion at a rate which may be too fast for the accretor to accept. The accreting star swells until the envelopes of both stars meet and become, quite literally, common.

What happens next is perhaps the most poorly understood process in binary stellar evolution, the common envelope phase (Paczyński 1976). This has never been observed directly but evidence in the form of very close binaries is plentiful. Close binaries with periods of one day or less and containing a carbon-oxygen white dwarf and a mainsequence star are well known. The cataclysmic variable systems are excellent examples (Warner 1995; Knigge 2011). Because the white dwarf is carbon rich it must have formed inside an AGB star which was hundreds of times the current size of the binary orbit. Some kind of magic converts a wide AGB-main-sequence binary into a close white-dwarfmain-sequence pair. The accepted view is that the wizardry is in the common envelope phase. 
Other examples of observed binary systems which have evolved through a common envelope phase include double neutron stars, AM CVn systems (Nelemans 2005) and close double white-dwarf systems such as those containing a helium white dwarf (Nelemans et al. 2005). Furthermore, single low-mass $\left(0.2 \mathrm{M}_{\odot}\right)$ helium white dwarfs can be formed by a common envelope around a low-mass giant and a planet (Nelemans \& Tauris 1998).

Some planetary nebulae, in which the final remnants of a stellar envelope are ionised by a hot, newly-formed white dwarf, may be formed by common envelope ejection (Yungelson et al. 1993; Soker 2006; Moe \& De Marco 2006). This evolutionary channel has many positive aspects which favour planetary nebula formation. These include rapid ejection of the stellar envelope, the emergence of a stripped stellar core to ionise the nebula and the formation of a binary system at the centre of the nebula. Many closes binary central stars of planetary nebulae have been found (De Marco et al. 2004). The immediate progenitors of these were likely to be the post-AGB stars (van Winckel et al. 2008).

Progress in understanding common envelope evolution in the last few years has focused on a few key problems, 1) three-dimensional modelling of the envelope ejection phase, 2) observational programmes to constrain the ejection efficiency and 3) population synthesis models of planetary nebula formation rates from common envelope ejection. We investigate the first two of these points. For details of the third please see Moe (this volume).

\section{The physics of common envelope ejection}

When a star ages it expands as a red giant. The internal structure is quite simple: a small core is surrounded by one or more burning shells above which lies a large convective envelope. In single-star evolution the envelope is eventually lost by stellar wind mass loss or, in stars with mass exceeding about $8 \mathrm{M}_{\odot}$, when the star explodes as a supernova. The core is then left exposed as a solitary white dwarf, neutron star or, in the most massive stars, a black hole .

In binary systems this relatively simple scenario is complicated by the presence of a companion (secondary) star (Figure 1). As the giant (primary) star expands, its outer layers are increasingly influenced by the gravitational field of the secondary star. When the envelope of the primary reaches the inner Lagrange point it is said to have filled its Roche lobe. Further expansion of the primary, which is inevitable because of stellar evolution, implies that material crosses the Lagrange point from the primary to the secondary and may become gravitationally bound to the secondary star (Paczyński 1971).

Roche-lobe overflow is often dynamically unstable because the primary has a convective envelope which expands upon mass loss (although Woods \& Ivanova 2011 and Passy et al. 2011 suggest this may not always happen). The mass-transfer rate then increases rapidly on a dynamical timescale of the primary star which is typically around one year for an AGB star. The transferred material falls onto the secondary star but can only be accreted stably at its thermal rate, which is orders of magnitude slower, so the secondary responds by expanding. Eventually the secondary star also grows to fill its Roche lobe: the stars then share a common envelope. Other forms of instability, such as the Darwin instability (conservation of angular momentum), also lead to common envelope (Iben \& Livio 1993).

The binary orbit decays inside the common envelope because of friction and a loss of co-rotation between the core and envelope (Podsiadlowski 2001). At first tidal forces drive the decay and it is slow (thousands of years) relative to the binary orbit (years). However as the stellar cores descend into ever denser material the drag, which is mostly gravitational rather than hydrodynamical (Ricker \& Taam 2012), increases and the rate of descent accelerates. This is the plunge phase. It occurs on a dynamical (or orbital) 
timescale of a few years. Tides are not sufficient to circularise the orbit in this phase so it becomes eccentric. Energy is lost from the orbit to the surrounding common envelope at a rate larger than the stellar luminosity. The envelope expands in response and some of it is unbound from the binary and ejected.

The ejection process naturally leads to a drop in the total mass and density of the common envelope and tides again synchronise the envelope with the orbit. The low density and reduction of shear reduce the drag force and rate of orbital energy loss such that the inspiral stalls. The drag force is small enough that the luminosity it generates can be radiated from the surface of the common envelope. Mass loss and inspiral continue but on a timescale much longer than the dynamical or orbital timescale. Other energy sources may contribute to envelope ejection including magnetic fields (Tout \& Regös 2003), recombination energy (Han et al. 1995; Dewi \& Tauris 2000) and nuclear burning (Ivanova \& Podsiadlowski 2002; Podsiadlowski et al. 2010).

The final fate of the binary system depends on whether the common envelope is ejected before the orbit becomes so small that the cores merge. The envelope must be ejected during the formation of systems such as cataclysmic variables and close double white dwarfs. It is less clear what happens when the cores merge. Perhaps a rapidly rotating FK Comae-type star or, in the case of a neutron star and a giant, a Thorne-Żytkow object is formed (Thorne \& Żytkow 1975)?

\section{Parameterised models of common envelope ejection}

Common envelope evolution is typically included in binary star population simulations by means of a parameterised model. The most commonly employed is the $\alpha$-formalism which treats the energy released during the inspiral phase as a fraction $\alpha$ of the change in orbital energy, $\Delta E_{\text {orb }}$, during the common envelope phase (Webbink 1984). Large values of $\alpha$ thus increase the likelihood of envelope ejection while small values often lead to coalescence and hence a single star. Population synthesis models often assume a constant $\alpha$ which is less than unity so that there is no energy generation during the inspiral. There are several subtly different definitions for $\alpha$ (see e.g. Davis et al. 2012; De Marco et al. 2011) and any population synthesis study must take care to be consistent. This also makes it difficult to compare values of $\alpha$ required to fit observations unless the prescription used is clearly stated (Section 5).

The orbital energy released is $\alpha \Delta E_{\text {orb }}$ and this is assumed to be available to expand and eject at least some of the envelope. The envelope binding energy must be known and the most common parameterisation is $E_{\mathrm{bind}}=G M_{\mathrm{env}} M_{\mathrm{c}} / \lambda R$ where $M_{\mathrm{env}}, M_{\mathrm{c}}$ and $R$ are the envelope mass, core mass and radius of the giant and $\lambda$ is a measure of central condensation of the star, a constant of order unity. The product $\alpha \lambda$ is then used to calculate the final separation of the binary on the assumption that the envelope is ejected. If $\alpha \lambda$ is small enough the cores overflow their Roche lobes and the system merges.

In principle $\lambda$ can be calculated from detailed stellar models (Dewi \& Tauris 2000; Loveridge et al. 2011; Xu \& Li 2010; Ivanova 2011) if we assume the common envelope structure is similar to the pre-Roche lobe overflow structure of the giant. Most population synthesis studies to date assume a constant value $\lambda=0.5$ or 1.0 . The calculation of $\lambda$ from stellar models is at present rather uncertain. The value of $\lambda$ can differ by an order of magnitude depending on the mass co-ordinate above which the envelope is ejected (Tauris \& Dewi 2001; Ivanova \& Chaichenets 2011). Post-ejection thermal readjustment of the stripped star may also be important (Ivanova 2011) but this is usually neglected. In evolved AGB stars it may be possible to tap the recombination energy of the envelope during the ejection process. If this is the case $\lambda$ may be much greater than 1 , perhaps up 


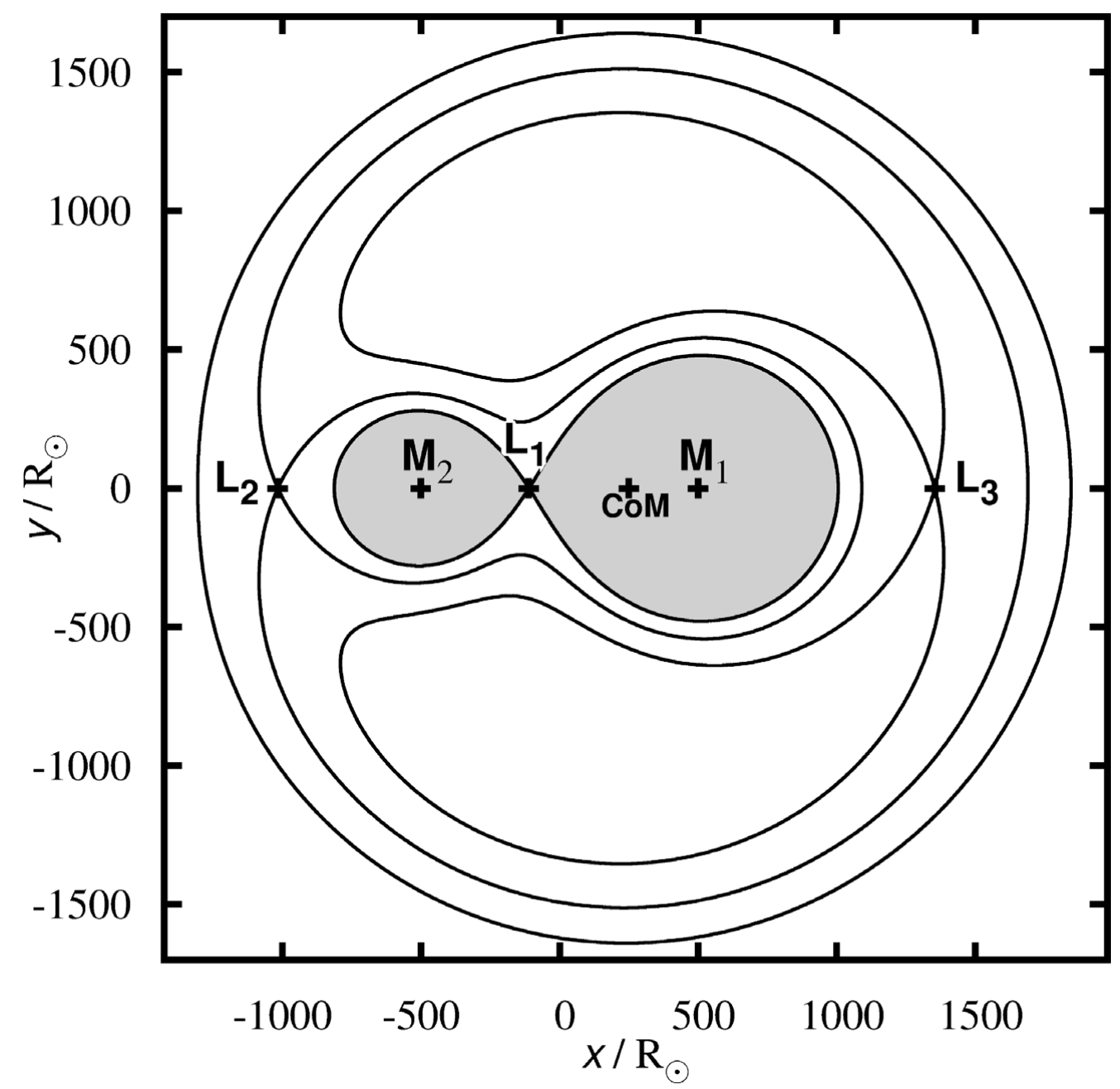

Figure 1. Equipotentials in the $x-y$ orbital plane of a binary with separation $1000 \mathrm{R}_{\odot}$ and component masses $M_{1}=1 \mathrm{M}_{\odot}$ and $M_{2}=1 / 3 \mathrm{M}_{\odot}$ centred at points $\mathbf{M}_{1}$ and $\mathbf{M}_{2}$ respectively. When the primary star fills its Roche lobe, it transfers material to the companion through the inner Lagrange point, marked $\mathbf{L}_{1}$. The secondary cannot accept the material and so also fills its Roche lobe (the lobes are filled in grey), co-rotation between the giant envelope and the dense cores is lost, and the stars enter common envelope evolution. Also shown are the second and third Lagrange points, $\mathbf{L}_{2}$ and $\mathbf{L}_{3}$ respectively, and the centre of mass $(\mathbf{C o M})$ of the binary system.

to 100 , because the envelope of the red giant is only marginally bound so the common envelope is easily ejected with little change in the orbital separation (Dewi \& Tauris 2000).

An alternative model is the $\gamma$-prescription of Nelemans et al. (2000). This parameterises the post-ejection binary separation in terms of the initial orbital angular momentum $J$ where $\gamma=\Delta J / J$ and $\Delta J$ is the change in the orbital angular momentum during common envelope ejection. A single value of $\gamma \approx 1.5$ well fits double white dwarf binaries (Nelemans \& Tout 2005). There has been criticism of the $\gamma$-prescription e.g. by Webbink (2008) and Woods et al. (2011). 


\section{Detailed models of common envelope ejection}

In recent years much progress has been made in detailed numerical modelling of common envelope evolution. Such models fall into two camps, full three-dimensional hydrodynamical models and one-dimensional hybrid stellar models. For the interested reader, an excellent review of 3D common envelope simulations is that of Taam \& Ricker (2010) while the review of Ivanova (2011) is also worth the effort.

Three dimensional models of common envelopes are hydrodynamical simulations which employ either smoothed-particle hydrodynamics (SPH) or grid-based methods (Sandquist et al. 1998, 2000; Ricker \& Taam 2008; Passy et al. 2011; Ricker \& Taam 2012). These simulations are ideal for modelling the dynamical plunge phase of common envelope evolution. Simulations start with an idealised scenario in which a main-sequence star, usually considered as a point mass because of its small size relative to the giant primary star, is inserted into a spherically symmetric primary. The amount of mass lost depends on many factors such as the initial mass ratio and numerical resolution (e.g. De Marco et al. 2003). Such models cannot follow the evolution much beyond a few dynamical or orbital timescales because physics not treated by the models, such as energy transport by convection, becomes important.

As an alternative to full three-dimensional modelling, one-dimensional models employ a stellar-evolution code to follow a particle on a shell as it moves into the red giant envelope (Podsiadlowski 2001). By definition these models cannot follow three-dimensional geometrical details of the envelope ejection and they must parameterise the drag force and frictional energy deposition (Meyer \& Meyer-Hofmeister 1979). However, what such models lack in multi-dimensional detail they make up for by the inclusion of physics such as energy transport (by radiation or convection)c which acts on timescales longer than the dynamical timescale. In one dimension it is possible to follow the common envelope ejection from the the initial mass transfer to the final ejection. Other details neglected in 3D, such as nuclear burning, are easily included and, in some cases, help ejection of the envelope (Podsiadlowski et al. 2010).

\section{Common envelope reconstruction and constraints on $\alpha$}

Three groups have recently attempted to constrain the efficiency of common envelope ejection. Given a close white-dwarf, main-sequence binary with a well-constrained white dwarf mass, the radius of the AGB parent star can be calculated because the core mass is monotonically related to its radius. From this, the initial binding energy is calculated (hence $\lambda$ ) and, because the stellar radius equals the Roche radius at the moment of mass transfer, the initial separation is also known. With this information, and the current stellar masses and orbital separation, the efficiency of common envelope ejection, $\alpha$, can be calculated.

At each stage in the process errors inevitably creep in. These accumulate into significant uncertainties, often spanning an order of magnitude, in the reconstructed $\alpha$ for each binary. Despite this, it is claimed that statistically significant correlations between $\alpha$ and the mass of the main-sequence star or the orbital period are found (Davis et al. 2012; De Marco et al. 2011) or are not found (Zorotovic et al. 2010). Why the results of these studies disagree is not clear. The samples used contain different stars and there may be technical details in the analyses that differ. What is apparent from these studies is that $\alpha$, for most of the sample binaries, lies between 0.1 and 1.0.

At present, no direct observations of stars in a common envelope phase have been reported. This does not mean that astronomers should stop looking. The recent merger 
of V1309 Sco demonstrates that human-timescale stellar-merger astronomy is possible (Tylenda et al. 2011). There are however candidates for very recent common envelope ejection. These include binary post-AGB stars with circumbinary discs (van Winckel et al. 2008) and planetary nebulae containing subdwarf-B stars (Geier et al. 2011).

\section{The common envelope family}

The ejection of a common envelope from an AGB star is of great interest in the study of planetary nebulae. Many planetary nebulae are asymmetric and, naturally, the search is on for the source of the asymmetry (see e.g. the proceedings of the - to date - five Asymmetric Planetary Nebulae conferences as well as Soker \& Livio 1994). Many planetary nebulae have close-binary central stars (De Marco et al. 2004) although it is not clear if presence of a companion star directly caused the envelope ejection. Miszalski et al. (2009) find a binary fraction of $10-20 \%$. Bi-polar nebulae are quite likely formed by common envelope ejection because material is ejected mostly around the equator of the envelope in the orbital plane.

Post-AGB stars are immediate progenitors of planetary nebulae and many are known be surrounded by circumbinary discs which presumably form from the envelope of their parent AGB star (de Ruyter et al. 2006). Such discs, if they are not evaporated quickly, may pump the orbital eccentricity in the binary as observed in the barium stars and influence the evolution of binary systems which lead to type Ia supernovae (Kashi \& Soker 2011).

Common envelope evolution also occurs in massive-star binaries (with primary masses greater than $8 \mathrm{M}_{\odot}$ ) for example when one star evolves into a red supergiant. After ejection of the common envelope the compact helium-burning core of the supergiant is left in a close binary with, typically, a main-sequence companion. The helium star explodes as a type $\mathrm{Ib} / \mathrm{c}$ supernova soon after it exhausts its helium fuel and the binary system, in some cases, survives the explosion as a neutron-star, main-sequence star binary. The main-sequence star itself then evolves until it overflows its Roche lobe. Material streams on to the neutron star releasing energy as X-rays and the system appears as an X-ray binary. The secondary, if massive enough, also explodes and this may lead to a double neutron star binary. In some binary stars a second common envelope phase ensues when the secondary also becomes a giant. As an example, all high-mass X-ray binaries will pass through a common envelope phase once Roche-lobe overflow is initiated. During the spiral-in phase the neutron star can merge with the helium core forming a Thorne-Żytkow object (Thorne \& Żytkow 1975). Taam \& Sandquist (2000) review common envelope evolution in massive binaries.

Merging in a common envelope has received some recent attention. The products of merging are all single stars and in some cases this can be used to identify populations of peculiar stars which may be post-common envelope mergers. The R-type carbon stars are all single and may arise from the merging of a giant star with a white dwarf companion (Izzard et al. 2007; Piersanti et al. 2010) while single sdB stars may merge in a common envelope (Politano et al. 2008). The more general case has been investigated by Politano et al. (2010) who predict the rotation rates of merged giants which can be compared to observations of e.g. FK Comae type stars.

\section{References}

Davis, P. J., Kolb, U., \& Knigge, C. 2012, MNRAS, 419, 287

De Marco, O., Bond, H. E., Harmer, D., \& Fleming, A. J. 2004, ApJL, 602, L93 
De Marco, O., Passy, J. C., Moe, M., Herwig, F., Mac Low, M. M., \& Paxton, B., 2011, MNRAS, 411,2277

De Marco, O., Sandquist, E. L., Mac Low, M. M., Herwig, F., \& Taam, R. E., 2003, Rev. Mexicana AyA-CS, 18,24

de Ruyter, S., van Winckel, H., Maas, T., Lloyd Evans, T., Waters, L. B. F. M., \& Dejonghe, H., 2006, $A \& A, 448,641$

Dewi, J. D. M. \& Tauris, T. M., 2000, A\&A, 360, 1043

Geier, S., Napiwotzki, R., Heber, U., \& Nelemans, G., 2011, A\&A, 528, L16

Han, Z., Podsiadlowski, P., \& Eggleton, P. P., 1995, MNRAS, 272,800

Iben, Jr., I., 1993, ApJ, 415, 767.

Iben, Jr., I. \& Livio, M., 1993, PASP, 105, 1373

Ivanova, N., 2011, Evolution of Compact Binaries, 447, 91

Ivanova, N., 2011, ApJ, 730, 76.

Ivanova, N. \& Chaichenets, S., 2011, ApJ, 731, L36.

Ivanova, N. \& Podsiadlowski, P., 2002, Ap\&SSS, 281, 191

Izzard, R. G., Jeffery, C. S., \& Lattanzio, J., 2007, A\&SA, 470, 661

Kashi, A. \& Soker, N., 2011, MNRAS, 417, 1466

Knigge, C., 2011, Evolution of Compact Binaries, 447, 3

Loveridge, A. J., van der Sluys, M. V., \& Kalogera, V., 2011, ApJ, 743, 49

Meyer, F. \& Meyer-Hofmeister, E., 1979, A\&SA, 78, 167

Miszalski, B., Acker, A., Moffat, A. F. J., Parker, Q. A., \& Udalski, A., 2009, A\&A, 496, 813

Moe, M. \& De Marco, O., 2006, ApJ, 650, 916

Nelemans, G., 2005, ASP-CS, 330, 27.

Nelemans, G., Napiwotzki, R., Karl, C., Marsh, T. R., Voss, B., Roelofs, G., Izzard, R. G., Montgomery, M., Reerink, T., Christlieb, N., \& Reimers, D., 2005, A $\xi A, 440,1087$

Nelemans, G. \& Tauris, T. M., 1998, A\&A A, 335, L85

Nelemans, G. \& Tout, C. A., 2005, MNRAS, 356, 753

Nelemans, G., Verbunt, F., Yungelson, L. R., \& Portegies Zwart, S. F., 2000, A\&A, 360, 1011

Paczyński, B., 1971, ARA\&A, 9, 183.

Paczyński, B., 1976, IAU Symp. 73: Structure and Evolution of Close Binary Systems, p. 75.

Passy, J. C., De Marco, O., Fryer, C. L., Herwig, F., Diehl, S., Oishi, J. S., Mac Low, M. M., Bryan, G. L., \& Rockefeller, G., 2011, Evolution of Compact Binaries, 447, 107

Passy, J. C., Herwig, F., \& Paxton, B., 2011, ArXiv e-prints, 1111.4202.

Piersanti, L., Cabezón, R. M., Zamora, O., Domínguez, I., García-Senz, D., Abia, C., \& Straniero, O., 2010, $A \mathscr{E} A, 522, \mathrm{~A} 80$.

Podsiadlowski, P., 2001, ASP-CS 229, 239.

Podsiadlowski, P., Ivanova, N., Justham, S., \& Rappaport, S., 2010, MNRAS, 406, 840

Politano, M., Taam, R. E., van der Sluys, M., \& Willems, B., 2008, ApJ, 687, L99

Politano, M., van der Sluys, M., Taam, R. E., \& Willems, B., 2010, ApJ, 720, 1752

Ricker, P. M. \& Taam, R. E., 2008, ApJ, 672, L41

Ricker, P. M. \& Taam, R. E., 2012, ApJ, 746, 74

Sandquist, E. L., Taam, R. E., \& Burkert, A., 2000, ApJ, 533, 984

Sandquist, E. L., Taam, R. E., Chen, X., Bodenheimer, P., \& Burkert, A., 1998, ApJ, 500, 909

Soker, N., 2006, ApJ, 645, L57

Soker, N. \& Livio, M., 1994, ApJ, 421, 219

Stancliffe, R. J., Chieffi, A., Lattanzio, J. C., \& Church, R. P., 2009, PASA, 26, 203

Taam, R. E. \& Ricker, P. M., 2010, New Astron. Revs, 54, 65

Taam, R. E. \& Sandquist, E. L., 2000, ARA\&A, 38, 113

Tauris, T. M. \& Dewi, J. D. M., 2001, $A \xi A, 369,170$

Thorne, K. S. \& Żytkow, A. N., 1975, ApJ, 199, L19

Tout, C. A. \& Regös, E., 2003, ASP-CS, 293, 100

Tylenda, R., Hajduk, M., Kamiński, T., Udalski, A., Soszyński, I., Szymański, M. K., Kubiak, M., Pietrzyński, G., Poleski, R., Wyrzykowski, Ł., \& Ulaczyk, K., 2011, AE\&A, 528, A114 
van Winckel, H., Deroo, P., Gielen, C., Reyniers, M., van Aarle, E., \& Vidal, E., 2008, AIP-CS, 1001, 349

Warner, B., 1995, Cambridge University Press.

Webbink, R. F., 1984, ApJ, 277, 355

Webbink, R. F., 2008, Ap\&SS, 352, 233.

Woods, T. E. \& Ivanova, N., 2011, ApJ, 739, L48.

Woods, T.E., Ivanova, N., van der Sluys, M. \& Chaichenets, S. Evolution of Compact Binaries, 447,127

Xu, X. J. \& Li, X. D., 2010, ApJ, 716, 114

Yungelson, L. R., Tutukov, A. V., \& Livio, M., 1993, ApJ, 418, 794.

Zorotovic, M., Schreiber, M. R., Gänsicke, B. T., \& Nebot Gómez-Morán, A., 2010, A\&\&A, 520, $\mathrm{A} 86$. 